\begin{tabular}{|c|l|}
\hline Title & Competition between spin-orbit interaction and Zeeman coupling in Rashba two-dimensional electron gases \\
\hline Author(s) & Meijer, F. E.; Morpurgo, A. F.; Klapwijk, T. M.; Koga, T.; Nitta, J. \\
\hline Citation & $\begin{array}{l}\text { Physical Review B, 70(201307(R)), 201307-1-201307-4 } \\
\text { https://doi.org/10.1103/PhysRevB.70.201307 }\end{array}$ \\
\hline Issue Date & 2004 \\
\hline Doc URL & http://hdl.handle.net/2115/14679 \\
\hline Rights & Copyright $\odot 2004$ A merican Physical Society \\
\hline Type & article \\
\hline File Information & PRB70-20.pdf \\
\hline
\end{tabular}

Instructions for use 


\title{
Competition between spin-orbit interaction and Zeeman coupling in Rashba two-dimensional electron gases
}

\author{
F. E. Meijer ${ }^{1, *}$ A. F. Morpurgo, ${ }^{1}$ T. M. Klapwijk, ${ }^{1}$ T. Koga,${ }^{2,3, \dagger}$ and J. Nitta ${ }^{2,4}$ \\ ${ }^{1}$ Kavli Institute of NanoScience, Delft University of Technology, Lorentzweg 1, 2628 CJ Delft, The Netherlands \\ ${ }^{2}$ NTT Basic Research Laboratories, NTT Corporation, Atsugi-shi, Kanagawa 243-0198, Japan \\ ${ }^{3}$ PRESTO-Japan Science and Technology Agency \\ ${ }^{4}$ CREST-Japan Science and Technology Agency
}

(Received 23 September 2004; published 22 November 2004)

\begin{abstract}
We systematically investigate how the interplay between the Rashba spin-orbit interaction and Zeeman coupling affects the electron transport and the spin dynamics in InGaAs-based 2D electron gases. From the quantitative analysis of the magnetoconductance, measured in the presence of an in-plane magnetic field, we conclude that this interplay results in a spin-induced breaking of time reversal symmetry and in an enhancement of the spin relaxation time. Both effects are due to a partial alignment of the electron spin along the applied magnetic field, and are found to be in excellent agreement with recent theoretical predictions.
\end{abstract}

DOI: $10.1103 /$ PhysRevB.70.201307

PACS number(s): 73.23.-b, 71.70.Ej, 72.25.Rb

Achieving control of the orbital motion of electrons by acting on their spin is a key concept in modern spintronics and is at the basis of many proposals in the field of quantum information. ${ }^{1}$ Two physical mechanisms are used to influence the dynamics of the electron spin in normal conductors: spinorbit interaction (SOI) and Zeeman coupling. In the presence of elastic scattering, these two mechanisms affect the spin in different ways. SOI is responsible for the randomization of the spin direction whereas the Zeeman coupling tends to align the spin along the applied magnetic field. Depending on the relative strength of these interactions, this interplay of SOI and Zeeman coupling is responsible for the occurrence of a variety of physical phenomena. ${ }^{2,3}$

Quantum wells (QW's) that define two-dimensional electron gases (2DEG's) are particularly suitable for the experimental investigation of the competition between SOI and Zeeman coupling, since they give control over many of the relevant physical parameters. Specifically, in these systems the SOI strength can be controlled by an appropriate QW design ${ }^{4}$ and by applying a voltage to a gate electrode. ${ }^{5,6}$ The electron mobility is usually density dependent, so that the elastic scattering time can also be tuned by acting on the gate. Finally, Zeeman coupling to the spin can be achieved with minimal coupling to the orbital motion of the electrons by applying a magnetic field parallel to the conduction plane.

In this Communication we study the competition of SOI and Zeeman coupling via magnetoconductance measurements in InGaAs-based 2DEG's with different Rashba SOI strength. From the detailed quantitative analysis of the weak antilocalization as a function of an applied in-plane magnetic field $\left(B_{\|}\right)$, we find that the partial alignment of the spin along $B_{\|}$results in a spin-induced time reversal symmetry (TRS) breaking, and in an increase of the spin relaxation time. The increase in spin relaxation time is found to be quadratic with $B_{\|}$, and strongly dependent on the SOI strength and the elastic scattering time. For both the spin-induced TRS breaking and the increase in spin relaxation time we find excellent quantitative agreement with recent theory. We also show that the quantitative analysis permits us to determine the in-plane $g$ factor of the electrons.

The three InAlAs/InGaAs/InAlAs quantum wells used in our work are very similar to those described in detail elsewhere. ${ }^{4}$ Here, we recall that each well is designed to have a different (Rashba) SOI strength. The characteristic spin-split energy $\Delta$ for the different samples is $\Delta \approx 0.51 .5$, and $1.8 \mathrm{meV}$ (in what follows we will refer to these samples as to samples 1,2 , and 3, respectively). The electron density and mobility at $V_{\text {gate }}=0 \mathrm{~V}$ are $n \simeq 7 \times 10^{15} \mathrm{~m}^{-2}$ and $\mu$ $\simeq 4 \mathrm{~m}^{2} / \mathrm{V} \mathrm{s}$. All measurements have been performed on $(20 \times 80 \mu \mathrm{m})$ Hall-bar shaped devices, at $1.6 \mathrm{~K}$. A $14 \mathrm{~T}$ superconducting magnet is used to generate $B_{\|}$and homemade split coils mounted on the sample holder are used to independently control the perpendicular field $\left(B_{\perp}\right)$. No significant difference in the results is observed when the in-plane field is applied parallel or perpendicular to the direction of current flow.

To understand how an in-plane magnetic field affects the electronic transport, we first discuss the behavior of sample 1 with the weakest SOI strength. Figure 1 shows the magnetoconductance of this sample measured as a function of $B_{\perp}{ }^{7}$ for different fixed values of the in-plane field $B_{\|}$. For small values of $B_{\|}$(main panel), the conductance exhibits a maximum at $B_{\perp}=0$, due to weak antilocalization (WAL) superimposed on the background of weak localization (WL). ${ }^{8}$ As $B_{\|}$ is increased, the amplitude of this maximum is reduced and eventually disappears around $B_{\|}=300 \mathrm{mT}$. A further increase in $B_{\|}$does not result in additional changes of the magnetoconductance until $B_{\|}$reaches approximately $1 \mathrm{~T}$ [Fig. 1(b)]. Upon increasing $B_{\|}$even further, the WL signal is also suppressed on the scale of several $(\simeq 10)$ T [Fig. 1(c)].

These observations allow us to conclude that the suppression of WAL and of WL in a parallel field are due to two distinct mechanisms causing time reversal symmetry breaking. At large fields, $B_{\|} \gg 1 \mathrm{~T}$, WL (which is not sensitive to the spin degree of freedom) is suppressed due to TRS breaking caused by the coupling of $B_{\|}$to the orbital motion of the electrons, owing to the finite thickness of the quantum well 


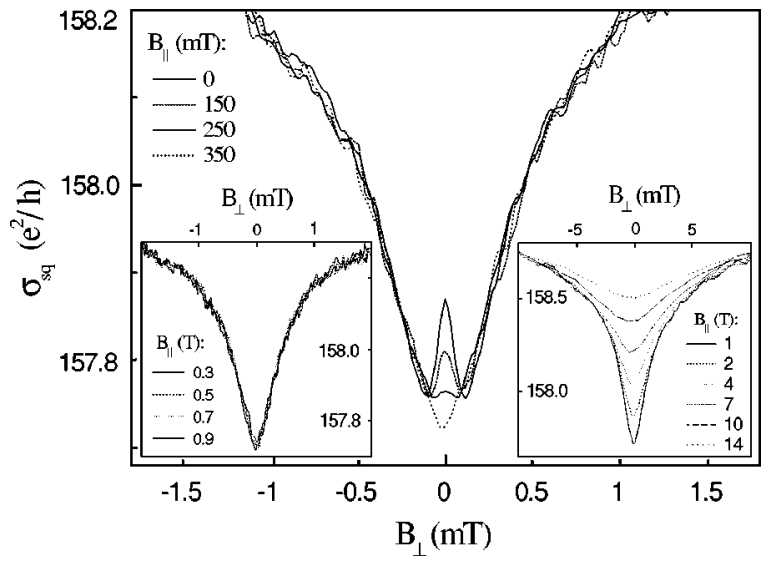

FIG. 1. The magnetoconductance $\sigma\left(B_{\perp}\right)$ of sample 1 at different values of $B_{\|}$. Three regimes can be identified: increasing $B_{\|}$from 0 to $350 \mathrm{mT}$ results in a suppression of the WAL peak (a), increasing $B_{\|}$further (up to about $B_{\|}=1 \mathrm{~T}$ ) does not induce additional changes in the $\sigma\left(B_{\perp}\right)$ curves (b), for values of $B_{\|}$larger than $1 \mathrm{~T}$ the WL is suppressed (c).

and the asymmetric confining potential. ${ }^{9}$ The suppression of the WAL peak at smaller values of $B_{\|}$originates from a spininduced TRS breaking due to the interplay between $B_{\|}$(Zeeman coupling) and SOI, as predicted theoretically. ${ }^{10}$ In this paper we will focus on the spin mechanism for TRS breaking, and discuss the orbital mechanism elsewhere.

The complete separation of spin and orbital TRS breaking, which is essential for the work presented here, has not been previously reported. ${ }^{3}$ In our samples, this separation is due to the small QW thickness $(\approx 10 \mathrm{~nm})$ and the small effective mass $\left(m^{*} \approx 0.041 m_{0}\right)$ which make the subband splitting in the QW relatively large, as well as to the relatively large gyromagnetic ratio $(g \simeq 3){ }^{9}{ }^{9,10}$ It allows us to account for the magnetoconductance curves $\sigma\left(B_{\perp}\right)$ measured at $B_{\|}<1 \mathrm{~T}$ in terms of existing theories that only consider the coupling of $B_{\|}$to the electron spin. Therefore, the number of parameters that need to be introduced for the quantitative analysis of the data is the smallest possible. This makes it possible to extract the values of the phase coherence time and the spin relaxation time as a function of $B_{\|}$with great accuracy, as it is needed to observe the dependence of $\tau_{s}$ on the in-plane magnetic field.

We have performed a quantitative analysis of the magnetoconductance curves on all samples and for different values of $n$ using the theory of Iordanskii, Lyanda-Geller, and Pikus (ILP), ${ }^{11}$ as it is appropriate for our samples, in which the spin relaxation is governed by the Dyakonov-Perel mechanism. ${ }^{12}$ Specifically, we have used Eq. (13) of Ref. 11 to fit the $\sigma\left(B_{\perp}\right)$ curves measured at different values of the in-plane field. In all cases, the elastic scattering time $\tau$ and the diffusion constant were determined by conventional longitudinal and Hall resistance measurements, and the Dresselhaus term was set to zero. ${ }^{4}$ Therefore, for each $n$ and $B_{\|}, \tau_{s}$ and $\tau_{\phi}$ are the only two remaining free fitting parameters. From this analysis, we find the $B_{\|}$dependence of $\tau_{s}$ and $\tau_{\phi}$, i.e., $\tau_{s}\left(B_{\|}\right)$ and $\tau_{\phi}\left(B_{\|}\right) \cdot{ }^{13}$

It is worth noting that in the ILP theory only one parameter is needed to account for the spin relaxation, since

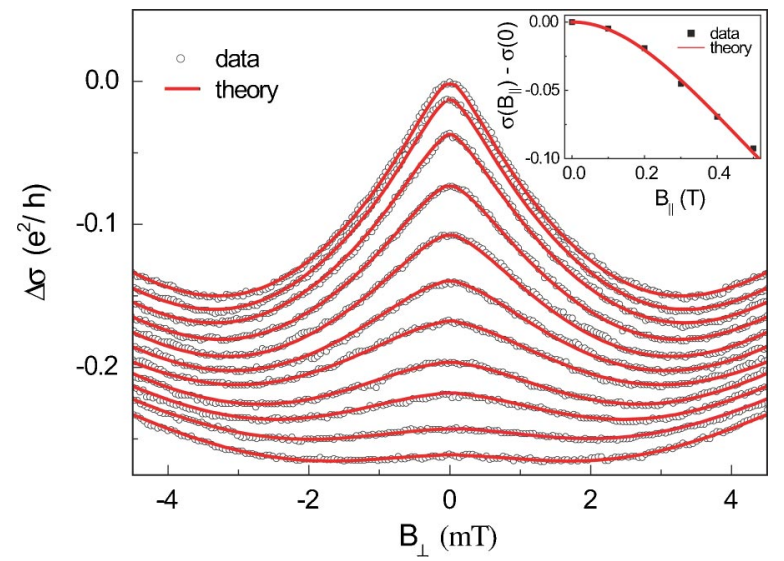

FIG. 2. The empty circles are the measured magnetoconductance $\Delta \sigma=\sigma\left(B_{\perp}\right)-\sigma(0)$ of sample 2 at different fixed values of $B_{\|}$ (offset for clarity). $B_{\|}$is increased from 0 to $1 \mathrm{~T}$, in steps of $0.1 \mathrm{~T}$ (top to bottom). The solid lines represent best fits to the ILP theory. The inset shows the amplitude of the WAL peak at $B_{\perp}=0$ as function of $B_{\|}$, i.e., $\sigma\left(B_{\perp}=0, B_{\|}\right)-\sigma(0,0)$, and the best fit to the theory (solid line).

$\tau_{s}(0) \equiv \tau_{s_{x}}(0)=\tau_{s_{y}}(0)=2 \tau_{s_{z}}(0)$. In the presence of an in-plane field, however, these relations may not hold, since relaxing the spin along $B_{\|}$costs energy $\left(\approx g \mu B_{\|}\right)$whereas relaxation in the direction perpendicular to $B_{\|}$does not. Nevertheless, for sufficiently small $B_{\|}\left(g \mu B_{\|}<\hbar / \tau_{s}(0), k T\right)$, the ratios between the different relaxation times are expected to change only minorly under the conditions of our experiments. This allows us to treat $\tau_{s}\left(B_{\|}\right)$as a single fitting parameter.

Figure 2(a) displays the results of the fitting procedure on sample 2 with the intermediate SOI strength. The continuous lines superimposed on the data represent the best fit to the ILP theory, and show that the agreement between data and theory is excellent for all values of $B_{\|}$. Similar agreement is obtained for the other samples and for all the different values of the electron density $n$. The values of $\tau_{\phi}\left(B_{\|}\right)$and $\tau_{s}\left(B_{\|}\right)$, as extracted from the fits, are shown in Figs. 3 and 4. Note that, since the electron mobility depends on the density, we are able to investigate how changing the elastic scattering $\tau$ affects the $B_{\|}$dependence of the phase coherence and of the spin-relaxation time. This is of particular interest as both $\tau_{\phi}\left(B_{\|}\right)$and $\tau_{s}\left(B_{\|}\right)$are predicted to depend on the DyakonovPerel spin relaxation time $\tau_{s}(0)$ [see Eqs. (2) and (3)], which is related to $\tau$ by the relation $1 / \tau_{s}(0)=\Delta^{2} \tau / 2 \hbar^{2}$. ${ }^{11}$

For all values of $n$, the measured $\tau_{\phi}\left(B_{\|}\right)$decreases as a function of $B_{\|}$(Fig. 3), which shows quantitatively the breaking of TRS due to the interplay of Zeeman coupling and SOI. This interplay is predicted to result in a quadratic dependence of $\tau_{\phi}$ on $B_{\|}:{ }^{10}$

$$
\frac{\tau_{\phi}\left(B_{\|}\right)}{\tau_{\phi}(0)}=\frac{1}{1+c B_{\|}^{2}},
$$

where $c$ is a constant given by

$$
c=\tau_{\phi}(0) \tau_{s}(0)\left(g_{\|}^{*} \mu_{B} / \hbar\right)^{2}
$$

and $g_{\|}^{*}$ is the in-plane $g$ factor. The solid lines in Fig. 3 are best fits to the data using Eq. (1) and treating $c$ as a (density 


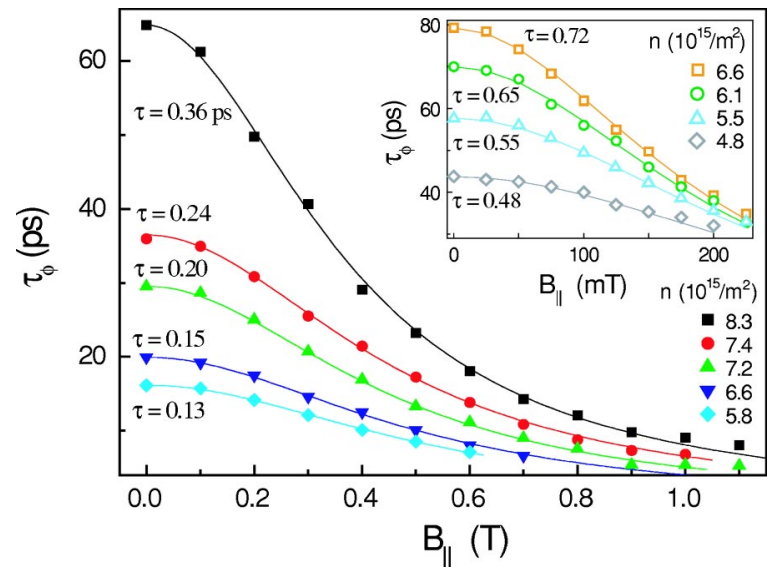

FIG. 3. (Color Online) The symbols represent $\tau_{\phi}$ as a function of $B_{\|}$, as extracted from the analysis of the magnetoconductance of sample 2, using the ILP theory (see Fig. 2). Different curves correspond to different values of $n$ (and elastic scattering time $\tau$ ). The solid lines are best fits based on the theory describing spin-induced dephasing (Ref. 10). The decrease of $\tau_{\phi}$ with decreasing electron density is consistent with dephasing originating from electronelectron interaction. The inset shows the extracted $\tau_{\phi}\left(B_{\|}\right)$and theoretical fits for sample 1.

dependent) fitting parameter. Also in this case the agreement between experiment and theory is excellent for all values of $n$ and for the different samples (the inset of Fig. 3 shows the behavior of sample 1; equally good agreement is found for sample 3).

Using the value of $c$ obtained from fitting the data of Fig. 3 we directly obtain $g_{\|}^{*}$ [Eq. (2)]. We find that, for each sample, the in-plane $g$ factor is approximately constant as a function of the electron density. The absolute values are determined to be $\left|g_{\|}^{*}\right|=2.8 \pm 0.1, \quad\left|g_{\|}^{*}\right|=3.3 \pm 0.1$, and $\left|g_{\|}^{*}\right|$

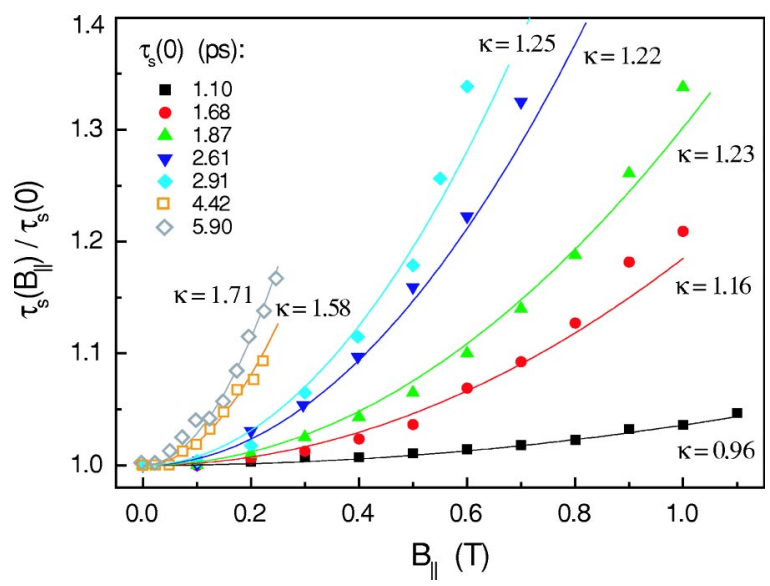

FIG. 4. (Color Online) The symbols represent $\tau_{s}$ as a function of $B_{\|}$, as extracted from the analysis of the magnetoconductance of sample 1 (open symbols) and sample 2 (filled symbols). Each set of symbols corresponds to a different value of the Dyakonov-Perel spin-relaxation time $\tau_{s}(0)$, with $1 / \tau_{s}(0)=\Delta^{2} \tau / 2 \hbar^{2}$ (controlled by changing the gate voltage). The solid lines are best fits to the theory [Eq. (3)], with $\kappa$ as an added parameter (see text). Note that the symbol code used in this figure for $\tau_{s}$ corresponds to that used in Fig. 3 for $\tau_{\phi}$.
$=3.5 \pm 0.1$, for samples 1,2 , and 3, respectively. Theoretically, the $g$ factor in our quantum well is predicted to depend substantially on its thickness, and is calculated to be $\left|g_{\|}^{*}\right|$ $=2.8$ and $\left|g_{\|}^{*}\right|=3.5$ for a thickness of 10 and $15 \mathrm{~nm}$, respectively. ${ }^{14}$ This agreement with theory gives additional support of our analysis in terms of spin-induced dephasing only, and shows that the measurement of WAL in the presence of an in-plane field permits to determine the in-plane $g$ factor. Contrary to other methods based on transport measurements, this method to determine the $g$ factor is suitable for disordered systems.

A different way to obtain $\tau_{\phi}\left(B_{\|}\right)$(and $c$ ), apart from fitting the whole $\sigma\left(B_{\perp}\right)$ curves measured at fixed $B_{\|}$, is by looking at the conductance at $B_{\perp}=0$ as function of $B_{\|}$. Specifically, the theory for spin-induced dephasing predicts that $\quad \sigma\left(B_{\perp}=0,0\right)-\sigma\left(B_{\perp}=0, B_{\|}\right)=\left(e^{2} / \pi h\right) \ln \left[\tau_{\phi}(0) / \tau_{\phi}\left(B_{\|}\right)\right]$ $=\left(e^{2} / \pi h\right) \ln \left(1+c B_{\|}^{2}\right) .{ }^{10}$ Also in this case, the agreement between theory and data is excellent (Fig. 2, inset) and the fitting procedure gives values for the parameter $c$ identical to those obtained above. This shows the consistency of our quantitative analysis and confirms once more the validity of the interpretation of the data in terms of spin-induced TRS breaking only.

Finally, Fig. 4 shows the behavior of the measured spin relaxation time as a function of $B_{\|}$for different densities and different strength of SOI interaction (samples 1 and 2). In all cases, the measured spin relaxation time increases quadratically with increasing the applied in-plane field. This directly shows that the presence of an in-plane field reduces spin randomization. The increase in $\tau_{s}\left(B_{\|}\right)$is more pronounced for a small strength of the SOI interaction and for short values of the elastic scattering time $\tau$, i.e., for long Dyakonov-Perel spin-relaxation times $\tau_{s}(0)$. This is because the Zeeman energy $g \mu B_{\|}$, that drives the alignment of the electron spin along $B_{\|}$, competes with the characteristic energy associated to the spin randomization $\hbar / \tau_{s}(0)$. Note that the opposite behavior, i.e., $\tau_{s}$ decreasing with increasing $B$, has been recently observed in systems with different spin-relaxation mechanisms. ${ }^{16}$

A quantitative analysis of the data requires a comparison with theory. For the case of a magnetic field normal to the conduction plane, extensive theoretical analysis exists. ${ }^{15}$ For the case of an in-plane field, in which the behavior of $\tau_{s}$ is determined by the Zeeman coupling and not by orbital effects, only the relaxation time of the $z$ component of the spin has been calculated as a function of $B_{\| \cdot}{ }^{17}$ When the Zeeman energy $g \mu B_{\|}$is much smaller than $\hbar / \tau_{s}(0)$, this quantity is given by

$$
\frac{\tau_{s_{z}}\left(B_{\|}\right)}{\tau_{s_{z}}(0)} \simeq 1+\frac{1}{2}\left[\kappa g_{\|}^{*} \mu_{B} B_{\|} \tau_{s}(0) / \hbar\right]^{2} .
$$

Although theoretical predictions for $\tau_{s_{x}}\left(B_{\|}\right)$and $\tau_{s_{y}}\left(B_{\|}\right)$are not available, we expect $\tau_{s_{x}}\left(B_{\|}\right) / \tau_{s_{x}}(0)$ and $\tau_{s_{y}}\left(B_{\|}\right) / \tau_{s_{y}}(0)$ to exhibit the same functional dependence as $\tau_{s_{z}}\left(B_{\|}\right) / \tau_{s_{z}}(0)$ as long as $g \mu B_{\|} \ll \hbar / \tau_{s}(0)$ and $k T$. This allows us to compare the measured $\tau_{s}\left(B_{\|}\right) / \tau_{s}(0)$ to Eq. (3). All the quantities that appear in Eq. (3) are known from the previous analysis, and 
we add a parameter $\kappa$ to achieve best fits to the data [theory ${ }^{17}$ predicts $\kappa=1$ in Eq. (3)]. Figure 4 shows that in all cases good agreement is obtained with $\kappa \simeq 1$ (continuous lines). We conclude that the qualitative behavior of the spinrelaxation time as a function of $B_{\|}, \tau$ and $\Delta$ [or, equivalently, $\left.\tau_{s}(0)\right]$ is the one expected, and that, within a small correction factor, our results are in quantitative agreement with theoretical predictions.

In view of the quantitative agreement between theory and data obtained throughout this work, it is worth considering the origin of the small correction factor $\kappa . \kappa \neq 1$ may originate from the limited accuracy with which the quantities in Eq. (3) are determined. The largest uncertainty comes from $g_{\|}^{*}$ and is approximately $10 \%$. An additional possibility is the $B_{\|}$-induced anisotropy of the in-plane spin relaxation times, i.e., $B_{\|}$breaks spin-rotational symmetry in the $2 \mathrm{D}$ plane. Although this anisotropy is expected to be small for $g_{\|}^{*} \mu B_{\|}$ $\ll \hbar / \tau_{s}(0)$ and $k_{B} T$, as mentioned before, it may result in a deviation from $\kappa=1$. Finally, for sample 1 with the weakest Rashba SOI, the Dresselhaus term may not be entirely negligible. ${ }^{18}$

In conclusion, we have observed how the partial alignment of the electron spin along an applied in-plane magnetic field determines the orbital and spin dynamics of electrons in Rashba 2DEG's. This alignment results in a spin-induced time reversal symmetry breaking and in a quadratic increase of the spin-relaxation time. The detailed quantitative analysis of our results demonstrates the validity of the existing theory and gives indications to its limits.

We would like to thank J. Schliemann, B. Nikolic, C.M. $\mathrm{Hu}$, and $\mathrm{H}$. Takayanagi for stimulating discussion and support. The work of AFM is part of the NWO Vernieuwingsimpuls 2000 program.
*Electronic address: f.e.meijer@tnw.tudelft.nl

†Present address: Graduate School of information Science and Technology, Hokkaido University, Sapporo, Japan.

${ }^{1}$ S. A. Wolf, D. D. Awschalom, R. A. Buhrman, J. M. Daughton, S. von Molnar, M. L. Roukes, A. Y. Chtchelkanova, and D. M. Treger, Science 294, 1488 (2001); D. D. Awschalom, D. Loss, and N. Samarth, Semiconductor Spintronics and Quantum Computation (Spinger-Verlag, Berlin, 2002); I. Zutic, J. Fabian, and S. Das Sarma, Rev. Mod. Phys. 76, 323 (2004).

${ }^{2}$ I. L. Aleiner and V. I. Falko, Phys. Rev. Lett. 87, 256801 (2001); R. Raimondi, M. Leadbeater, P. Schwab, E. Caroti, and C. Castellani, Phys. Rev. B 64, 235110 (2001); B. I. Halperin, A. Stern, Y. Oreg, J. N. H. J. Cremers, J. A. Folk, and C. M. Marcus, Phys. Rev. Lett. 86, 2106 (2001); M. Governale, ibid. 89, 206802 (2002); J. H. Cremers, P. W. Brouwer, and V. I. Falko, Phys. Rev. B 68, 125329 (2003); J. Schliemann and D. Loss, ibid. 68, 165311 (2003); K. Held, E. Eisenberg, and B. L. Altshuler, Phys. Rev. Lett. 90, 106802 (2003).

${ }^{3}$ D. M. Zumbuhl, J. B. Miller, C. M. Marcus, K. Campman, and A. C. Gossard, Phys. Rev. Lett. 89, 276803 (2002); G. M. Minkov, A. V. Germanenko, O. E. Rut, A. A. Sherstobitov, L. E. Golub, B. N. Zvonkov, and M. Willander, cond-mat/0312074 (unpublished).

${ }^{4}$ T. Koga, J. Nitta, T. Akazaki, and H. Takayanagi, Phys. Rev. Lett. 89, 046801 (2002).

${ }^{5}$ J. Nitta, T. Akazaki, H. Takayanagi, and T. Enoki, Phys. Rev. Lett. 78, 1335 (1997); G. Engels, J. Lange, T. Schapers, and H. Luth, Phys. Rev. B 55, R1958 (1997); T. Schapers, J. Appenzeller, H. Hardtdegen, and H. Luth, J. Appl. Phys. 83, 4324 (1998); D. Grundler, Phys. Rev. Lett. 84, 6074 (2000).

${ }^{6}$ Yu. A. Bychkov and E. I. Rashba, J. Phys. C 17, 6093 (1984).

${ }^{7}$ Experimentally, the value of $B_{\perp}$ is obtained from the measurement of the Hall voltage performed simultaneously to the mea- surement of the longitudinal conductivity. In the presence of a large $B_{\|}$, this automatically takes into account any small misalignment of the sample.

${ }^{8}$ For an anaysis of WAL at $B_{\|}=0$, see, e.g., W. Knap, C. Skierbiszewski, A. Zduniak, E. Litwin-Staszewska, D. Bertho, F. Kobbi, J. L. Robert, G. E. Pikus, F. G. Pikus, S. V. Iordanskii, V. Mosser, K. Zekentes, and Yu. B. Lyanda-Geller, Phys. Rev. B 53, 3912 (1996); T. Hassenkam, S. Pedersen, K. Baklanov, A. Kristensen, C. B. Sorensen, P. E. Lindelof, F. G. Pikus, and G. E. Pikus, ibid. 55, 9298 (1997); A. Zduniak, M. I. Dyakonov, and W. Knap, ibid. 56, 1996 (1997).

${ }^{9}$ H. Mathur and H. U. Baranger, Phys. Rev. B 64, 235325 (2001); J. S. Meyer, A. Altland, and B. L. Altshuler, Phys. Rev. Lett. 89, 206601 (2002); V. I. Fal'ko and T. Jungwirth, Phys. Rev. B 65, 081306(R) (2002).

${ }^{10}$ A. G. Malshukov, K. A. Chao, and M. Willander, Phys. Rev. B 56, 6436 (1997).

${ }^{11}$ S. V. Iordanskii, Y. B. Lyanda-Geller, and G. E. Pikus, Pis'ma Zh. Eksp. Teor. Fiz. 60, 199 (1994) [JETP Lett. 60, 206 (1994)].

${ }^{12}$ M. I. Dyakonov and V. I. Perel, Sov. Phys. JETP 33, 1053 (1971); Sov. Phys. Solid State 13, 3023 (1972).

${ }^{13} \tau_{\phi}\left(B_{\|}\right)$is the upper cutoff time for interference of time-reversed paths [also denoted as $\tau_{H}$ or $\tau_{B_{\|}}$in literature (see, e.g., Refs. 9 and 10)].

${ }^{14} \mathrm{R}$. Winkler, Spin-Orbit Coupling Effects in Two-Dimenional Electron and Hole Systems (Springer-Verlag, Berlin, 2003), p. 133.

${ }^{15}$ For most recent work see A. A. Burkov and L. Balents, Phys. Rev. B 69, 245312 (2004), and references therein.

${ }^{16}$ J. M. Kikkawa and D. D. Awschalom, Phys. Rev. Lett. 80, 4313 (1998); R. Hanson (private communication).

${ }^{17}$ V. A. Froltsov, Phys. Rev. B 64, 045311 (2001).

${ }^{18}$ N. S. Averkiev and L. E. Golub, Phys. Rev. B 60, 15582 (1999). 